\title{
Gender role attitudes and male-female income differences in China
}

\author{
Shisong Qing(D)
}

\author{
Correspondence: ssqing@soci.ecnu. \\ edu.cn \\ School of Social Development and \\ The Center for Modern Chinese City \\ Studies, East China Normal \\ University, 500 Dongchuan Ave, \\ Shanghai 200241, China
}

\begin{abstract}
By investigating deep-rooted cultural norms, this paper explores whether and how traditional gender role attitudes impact income gaps between men and women and identifies causal effects via instrumental variable and other causal inference methods. Based on data from the Chinese General Social Survey in 2013, the results show that traditional gender role attitudes have a strong negative effect to the earnings of women but have no significant effect on men's incomes. Through Oaxaca-Blinder decomposition, this research finds that the different effects of gender role attitudes on the incomes of men and women appear to play a prominent role in causing the gender gap in earnings. In addition, gender role attitudes have an indirect and broad effect on gender income inequality through educational attainment, labor force participation, working hours, and occupational status. These results provide us with a new perspective for understanding the persistence and mechanisms of gender income stratification under educational equalization and have implications for gender equality policies.
\end{abstract}

Keywords: Income, Gender income gap, Gender role attitudes, Cultural norms, Labor Force Participation, Gender discrimination

\section{Introduction}

Gender inequality in China has drawn widespread concern in the context of social system transition and structural change. Along with fertility decline and educational development, new generations of women are achieving increasing levels of education, implying a trend toward educational gender equalization. This trend seems to indicate substantial advancements in women's socioeconomic status and a narrowing of the income gap between men and women. It is perplexing, however, to find that the gender income gap is not only persistent but also widening (see Li et al. 2014). Motivated by this paradoxical phenomenon, researchers have conducted numerous studies on this trend.

The classical narrative method emphasizes the fundamental explanation for human capital and gender discrimination. ${ }^{1}$ A large amount of research conforming to the

\footnotetext{
${ }^{1}$ Gender segregation (by occupation, industry, and department) is a central mechanism of gender stratification. Yet, gender segregation can be theoretically explained by human capital and gender discrimination.
}

(c) The Author(s). 2020 Open Access This article is licensed under a Creative Commons Attribution 4.0 International License, which permits use, sharing, adaptation, distribution and reproduction in any medium or format, as long as you give appropriate credit to the original author(s) and the source, provide a link to the Creative Commons licence, and indicate if changes were made. The images or other third party material in this article are included in the article's Creative Commons licence, unless indicated otherwise in a credit line to the material. If material is not included in the article's Creative Commons licence and your intended use is not permitted by statutory regulation or exceeds the permitted use, you will need to obtain permission directly from the copyright holder. To view a copy of this licence, visit http://creativecommons.org/licenses/by/4.0/. 
analytical framework attributes the gender income gap to characteristics and inconsistent market returns of productivity. The former is regarded as "reasonable" while the latter is condemned as an "unreasonable" factor demonstrating that gender discrimination prevails in labor markets (e.g., prejudice and statistical discrimination). ${ }^{2}$ Many studies explain changing trends and causes of the gender income gap by analyzing how economic development, marketization, or distribution system reform affect human capital mechanisms or gender discrimination, although they seek macro-level explanations (Li and Li 2008; He and Wu 2015; Wang et al. 2008).

Existing paradigms and findings have laid solid foundations for understanding the formation mechanisms and changing trends of the gender income gap. However, challenges and dilemmas of theoretical and empirical analysis remain. The most controversial question posed in the theoretical arena concerns whether gender differences in characteristics of productivity (if present) originate in biological differences, rational choices made by individuals, or unreasonable social cultures and institutions. Differences in productivity characteristics between men and women, which are used to explain the gender income gap, require further "explanation," as neither men nor women were born or grew up in a gender-neutral cultural environment through their socialization (Lips 2013). For instance, some studies suggest that patriarchy or traditional gender role attitudes substantially lead to educational inequality between men and women, especially in rural areas (Wu 2012). In this sense, gender differences in human capital, if existent, cannot fully explain the gender income gap.

What challenges empirical analysis is the fact that the majority of the gender income gap cannot be explained even with the inclusion and control of human capital, occupations, labor markets, family structures, and other observable variables. For example, Blau and Kahn (2017) find that traditional explanatory variables such as human capital have limited and declining explanatory power in explaining the gender income gap in the United States. Chinese data also demonstrate the rather limited explanatory power of human capital and occupational segregation (Wu and Wu 2009). It is reasonable to ask whether some important factors have been neglected and how impact mechanisms are generated. Both theoretical problems and empirical challenges imply a need for new theory construction, which is the goal of this paper, to clarify the stratification of gender income and propose new strategies.

Two refined approaches are emerging from studies of the gender income gap. First, more features of labor suppliers should be considered in estimations, including personality traits ${ }^{3}$, psychological characteristics, risk preferences, and sense of competition, to develop the implications of human capital theory and enhance its capacity to explain the gender income gap (Zheng and Qing 2016). While studies have developed the logical interpretations of human capital theory, they still cannot provide a convincing explanation for whether personality traits or psychological characteristics are socially constructed, i.e., whether they are inherent or culturally constructed. Second, some new studies emphasize institutional and cultural impacts on gender inequality in

\footnotetext{
${ }^{2}$ Unobservable and uncontrollable features of productivity are those that cannot be explained by productivity. It is unreasonable to group all of these under the scope of gender discrimination. Therefore, refined knowledge of impact factors and formation mechanisms of the gender income gap relies on an exploration of new explanatory variables.

${ }^{3}$ Personality traits are non-cognitive skills rather than cognitive skills, which are considered human capital factors with economic return.
} 
exploring the social origins of gender behavior patterns and the income gap. Sociologists, feminist scholars, and economists have noted the potential influences of preferences, cultural norms, and institutional factors on gender inequality for a long time. However, direct evidence remains limited due to difficulties with concept definition and measurement. In recent years, psychological characteristics such as motivation, attitudes, expectations, and especially socially constructed gender role attitudes have been operationally measured ${ }^{4}$ and utilized to explore the gender income gap in empirical studies (see Bertrand 2011).

In fact, gender inequality is deeply rooted in traditional cultural norms (Jayachandran 2015). Some recent research finds that gender role attitudes originating from plowing agricultural systems have been persistent for thousands of years and influential in shaping gender inequality in modern labor settings (Alesina et al. 2013; Hansen et al. 2015). This finding demonstrates that traditional gender role attitudes exhibit undoubted continuity and have had persistent effects through their evolution. The profound influence of patriarchy on gender inequality in China is worthy of exploring given the country's several thousand-year-old agricultural civilization and Confucian tradition. Moreover, the gender discourse has drastically changed in Chinese society since the country's market reformation and institutional transition (Wu 2009). Official discourses of gender, e.g., "women are as good as men" and "women hold up half the sky," have lost their institutional basis little by little. In the wave of market expansion and the era of media, gender role attitudes, especially those of the social division of labor, have been restored to their traditional form (Jia and Ma 2015; Yang 2017). These changes provide unique cases for exploring whether and how gender role attitudes impact the gender income gap and offer a key opportunity to further the theory of gender stratification. Unfortunately, existing works on gender income stratification in China have not empirically analyzed or tested this theoretical logic. The extent to which gender role attitudes affect the gender income gap remains vaguely determined in academia, not to mention how this impact develops.

Therefore, inspired by a social-cultural perspective, this paper follows and furthers this evolution by considering whether and how traditional gender role attitudes affect the gender income gap between men and women. The significance of this paper is based in its use of two perspectives. First, the new perspective of social culture is utilized to explore the cause of the gender income gap. In addition to education, working hours, and occupation, this paper brings gender role attitudes into the discussion of the gender income gap as a new explanatory factor. Second, this paper focuses on the impact mechanisms of gender role attitudes by scrutinizing how gender role attitudes impact the gender income gap through productivity characteristics such as human capital; this paper in turn extends beyond limited discussions focused on the labor market and exposes the social-cultural roots of productivity characteristics and the gender income gap. In sum, this paper represents an endeavor to deepen and further the explanatory framework of the gender income gap, to provide a new perspective in

${ }^{4}$ Gender role attitudes refer to individual recognition of gender role norms and behavior patterns, which are measured from individual opinions towards the gendered division of labor in markets and households and typologically defined as traditional and modern gender role attitudes. Some Chinese researchers have used the term gender attitudes for short. Other alternative terms include gender ideology, gender egalitarianism, and gender identity. 
examining and interpreting the gender income gap and its persistence and to serve as a practical reference for ways of mitigating gender income discrepancies to promote gender equality and women's development.

This paper elaborates on the theoretical link between gender role attitudes and the gender income gap, establishes an analytical framework, and proposes research hypotheses. An empirical analysis is conducted based on a nationally representative data sample. Potential selection biases of the data sample are fixed in the process of empirical testing and data analysis. Instrumental variable estimation and other causal inference methods are utilized to draw valid research conclusions with convincing research significance and constructive policy implications.

\section{Literature review and hypotheses}

\section{Theory and literature review}

Gender role-related cultural norms provide a vital theoretical perspective in the explanation of gender inequality. Social role theory (Eagly 1987) or gender role theory (Gutek et al. 1991) suggests that gender role expectations and norms socially imposed on both men and women constitute the basis for social behavioral differences between men and women. Traditional cultural norms and gender stereotypes collectively work to link women to family roles whereby society expects women to concentrate on housework or engage in traditional feminine occupations and master associated skills. Men are expected to support their families economically and improve upon their abilities to access market resources. Therefore, cultural gender values are prominent forces resulting in different investments in human capital, differences in capacity, and an income gap between men and women by emphasizing normative gender roles for men and women and encouraging them to invest in different domains. These cultural values have unobtrusively been applied to children's socialization, internalized into children's subjective consciousness, and passed down from generation to generation. These cultural values are unconsciously exercised in societal life, strengthening and reproducing gender roles and gender structures (Eagly and Wood 1999). Economic studies on the utility analysis of identity have made similar propositions. Drawing on sociological and psychological concepts, Akerlof and Kranton (2000) point out that identity and self-image have direct impacts on individual utility, as individuals' belonging to social categories (not limited to gender) and recognition of group behavioral norms constitute their motivation bases for daily life and social interactions. Deviating from certain group behavioral norms reduces one's utility and can even expose one to prejudice and punishment. As a basic social category, gender implies the presence of different role expectations and behavioral norms between men and women, and this presence maintains and strengthens gender identification by encouraging convergence and excluding heterogeneity. In this sense, gender identity has a direct impact on individual behavior and labor market outcomes.

Empirical evidence supports the sociocultural attributes of gender role attitudes and their influence on gender structures. A large amount of literature finds that gender role attitudes have a significantly negative impact on income and especially on women's incomes. Corrigall and Konrad (2007), for instance, in analyzing tracing data on American high school students, find that traditional gender role attitudes held by individuals in their teens reduce women's working hours and hourly wage rates in paid jobs yet do not 
significantly affect men's working hours and incomes. Furthermore, gender role attitudes have nothing to do with the incomes of white men, although it has been verified that the influence of gender role attitudes on income is moderated by ethnic identities, whereby traditional gender role attitudes have more negative impacts on white women (or white mothers) than on black women (mothers) in terms of income (Christie-Mizell 2006; Christie-Mizell et al. 2007). It is worth noting that Stickney and Konard (2007) empirically verify the universality of the relation between traditional gender role attitudes and low incomes among women by studying social survey data from 28 countries and regions. Moreover, Judge and Livingston (2008) find a negative relation between traditional gender role attitudes and women's incomes and a positive relation between traditional gender role attitudes and men's incomes from data from the National Longitudinal Survey of Youth in the US for 1979 to 2004. Therefore, under both positive and negative effects, traditional gender role attitudes are bound to widen the income gap between men and women.

It is worth noting that while the majority of related research has been conducted in Western countries, theoretical propositions made can logically apply to Chinese society. The ontology of Chinese traditional thoughts of Yin and Yang and the view that "man is superior to woman" engender gender relations embedded in spheres of politics, economy, society, and family. However, China has also launched multidimensional and instrumental women's liberation movements from top to bottom to unprecedentedly remold traditional gender cultures since the May Fourth New Culture Movement. Especially in the 1950s, "de-gendering" strategies have blurred or eclipsed gender differences through an emphasis on gender equality based on "social obligations and contributions." These trends created new forms of gender inequality and especially in the family, in which traditional gendered divisions of labor and patriarchal cultural characteristics have been preserved (Song 2012; Zuo 2005). Through the country's reform and opening-up process and market transition, "tradition restoration" has been observed in gender role attitudes and gendered divisions of labor with the abolishment of the unit welfare system and the decline of official gender equality institutions.

Under such conditions, empirical research conducted on Chinese society verifies that traditional gender culture has a consistent influence in both the public and private spheres. For example, it has been found that gender role attitudes have significant effects on the gendered division of chores (Tong and Liu 2015; Yang 2014). Influenced by traditional attitudes, women are inclined toward "gender performance" whereby as their housework hours increase, their relative income status is elevated to a certain degree (Liu et al. 2015). Meanwhile, traditional gender role attitudes have no significant influence on men's labor force participation while hindering and repressing women's labor force participation (Qing 2017). Modern gender role attitudes of equality facilitate women's career development.

In sum, the potential relation between gender role attitudes and the gender income gap is universal. Moreover, cultural norms have remained influential throughout China's transitional period. From this basic statement, this paper further develops an analytical framework to not only reveal the influence of gender role attitudes on individual incomes but also explore their effecting mechanisms. The paper also supplements and develops studies of gender inequality focused on China's specific social context by focusing on the income gap between men and women under the restoration of traditional Chinese gender role attitudes. 


\section{Hypotheses and analytical framework}

Existing theories and empirical studies demonstrate that cultural norms have shaped gendered differences in behavior and have resulted in unequal consequences through different behavior patterns. Furthermore, gendered cultural norms form a set of typical double standards that place more severe restrictions on women in terms of compliance with norms and punishments for deviance. Therefore, we can observe women's "gender performance" outside of spontaneous behaviors. It is thus reasonable to assume that gendered cultural norms primarily regulate and restrain women's daily actions and their domains of social activity in their interactions with other individuals, with their families and in society while supporting the family is defined as men's indispensable responsibility and obligation. Hence, gender role attitudes are posited to have different influences on men's and women's incomes based on existing theories and empirical studies. This paper thus hypothesizes that as follows:

Hypothesis 1: The influence of gender role attitudes on income is moderated by gender identity whereby gender role attitudes primarily affect women's incomes. Ceteris paribus, equal gender role attitudes have positive impacts on women's incomes while traditional gender role attitudes restrain women's incomes.

If hypothesis 1 is verified, it can be inferred that gender role attitudes have direct impacts on the gender income gap and that traditional gender role attitudes impact the gender income gap by decreasing women's incomes rather than by increasing men's incomes. However, hypothesis 1 does not imply that women who hold traditional gender attitudes should have rather low incomes. In contrast, as discussed above, it is problematic that women with traditional gender attitudes have negative impacts on income, reflecting regulations, and constraints of social culture placed on women, as women's gender role attitudes and corresponding behavior patterns are shaped by specific social cultural environments.

In this sense, gender role attitudes formed in early stages of life affect not only career choices and incomes in the labor market but also traditional explanatory factors of the gender income gap, such as educational attainment, labor force participation, and time dedicated to work and family. Therefore, Firestone et al. (1999) claim that when human capital and other variables are controlled in the model, the direct impacts of gender role attitudes on income cannot represent all of their effects. In other words, exploring the influence of social cultural factors, e.g., gender role attitudes, on the gender income gap cannot be accomplished by adding new variables. This paper thus develops Judge and Livingston's (2008) analytical framework of mediating mechanisms of career choice ${ }^{5}$ and elaborates on the indirect influence of gender role attitudes on incomes and the gender gap from three empirical mechanisms or approaches. This paper thus proposes hypothesis 2 on mediating mechanisms.

\footnotetext{
${ }^{5}$ They bring impact factors of gender role attitudes into the general analytical framework. This paper primarily focuses on the social economic consequences of gender role attitudes, which constitute the influencing mechanisms of income and gender inequality.
} 
Hypothesis 2: The indirect impact of gender role attitudes on the gender income gap is mediated by educational attainment, labor force participation, working hours and occupational status.

Educational attainment serves as a vital means through which gender role attitudes can affect gender inequality in the labor market. Traditional gender role attitudes define men as breadwinners and establish a gendered division of labor of "breadwinning men and homemaking women." Motivations of human capital investment and accumulation for women are relatively insufficient, further affecting the gender gap in human capital. For instance, a longitudinal survey conducted in the US finds that middle school students with traditional gender role attitudes, e.g., "breadwinning men and homemaking women," and especially female students with such views not only exhibit relatively low educational expectations (Davis and Pearce 2007) but also receive less formal education and even less on-the-job training upon entering the labor market (Kosteas 2013). Due to the traditional view that "women are inferior to men" and specifically that women are believed to be less proficient in mathematics than men, boys and girls enter different educational channels starting from high school (van der Vleuten et al. 2016). Therefore, it is not difficult to conclude that traditional gender role attitudes form and influence the gender income gap in the labor market from early stages of the life course through their impacts on educational attainment. We thus propose our second hypothesis of mediating mechanisms.

Hypothesis 2a: Equal gender role attitudes have a positive effect on women's educational attainment while traditional gender role attitudes hinder women's access to education and indirectly affect their incomes.

Gender role attitudes also influence gender segregation and income inequality through occupational preferences or values. In line with previous research on educational attainment, traditional gender role attitudes not only affect the choice of education majors but also further shape career expectations corresponding to a specific gender and capacity (Correll 2004). At the same time, gender role attitudes shape the different occupational preferences and values of men and women (Sinclair and Carlsson 2013). It has been found that women with traditional gender role attitudes value interpersonal relationships and feelings of social altruism achieved through work while men seek material rewards, prestige, and status from their work (Diekman et al. 2010). As a result, men and women enter the labor market with different expectations and preferences, choose different occupations, and thus contribute to gendered segregation and the income gap (Okamoto and England 1999). In addition, traditional gender role attitudes and cultural norms hinder women's access to management positions and especially to senior positions (Haveman and Beresford 2012). This hindrance is mainly the case because the female image perceived and recognized by society is not consistent with the roles of managers. "Female managers" may encounter social prejudice (Eagle and Karau 2002). A recent experimental study also shows that unmarried women deliberately limit their career pursuits to achieve an image of a "good wife" in men's minds (Bursztyn et al. 2017), perpetuating vertical gender segregation and the income gap in terms of positions. Therefore, to analyze the comprehensive impacts of gender role attitudes on horizontal 
occupational segregation and vertical position segregation, we propose the following hypothesis on mediating mechanisms.

Hypothesis 2b: Equal gender role attitudes have a positive effect on women's career development while traditional gender role attitudes hinder women's acquisition of occupational status and indirectly affect their incomes.

Labor force participation and working hours serve as another channel through which gender role attitudes affect the income gap between men and women. The traditional patriarchy and gender role attitudes emphasize the difference between men and women in reproductive functions and family care capacities, which force women to undertake unpaid chores. This pattern of the gendered division of labor does not change even when women have more resources or relatively higher status. Studies have found that when women earn more than their husbands, they do even more chores to compensate for their deviation from traditional cultural norms in the labor market (Bertrand et al. 2015; Liu et al. 2015). Gender inequality in the private sphere inevitably permeates and affects the public sphere and market. To reserve time and energy for family responsibilities, women with traditional gender role attitudes often reduce their working hours in the labor market despite entering the labor market for paid work (Corrigall and Konrad 2007). Some even withdraw from the labor market to assume family roles (Fortin 2015). Therefore, traditional cultural norms affect both power relationships in the private sphere and resource allocation in the public sphere and force women to accept unequal conditions either actively or passively. We thus propose the following hypothesis on mediating mechanisms.

Hypothesis 2c: Equal gender role attitudes have a positive effect on women's labor force participation while traditional gender role attitudes hinder women's labor force participation and reduce their working hours and indirectly affect their incomes.

Based on the above theoretical analysis and research hypotheses, a theoretical framework and diagram of variables are constructed (see Fig. 1). As shown in Fig. 1, gender

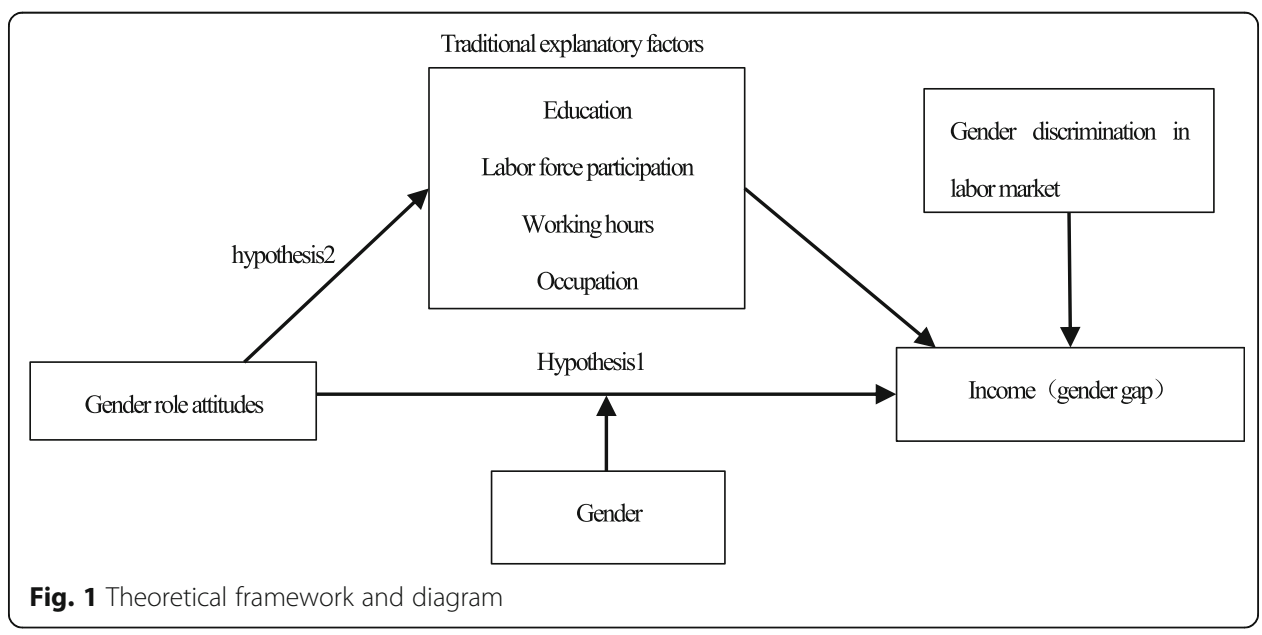


role attitudes have a direct impact on income. This impact may be moderated by gender identity, which mainly affects the behavior patterns and incomes of women (hypothesis 1). At the same time, gender role attitudes developed in childhood or adolescence can also indirectly affect the income and gender income gap through mediating channels such as education attainment, labor force participation and working hours, and career selection and promotion (hypothesis 2). In addition, when controlling other factors, we must recognize that in terms of employment, wage payments, and job promotion, gender discrimination is central to explaining the lower incomes of women. Therefore, the theory of gender discrimination is included in the analysis framework adopted in this work.

Figure 1 demonstrates that the analysis paradigm applied in this work extends beyond the limitations of human capital and gender discrimination to a comprehensive analysis framework based on a new perspective and integration of multiple theories. Among them, productivity variables such as human capital are no longer regarded as predetermined variables relating to "staying out of business" and are instead viewed as influenced by relatively stable gender role attitudes formed in early stages of the life course, although human capital remains as the basic theory. This paper represents an endeavor to deepen understanding of the gender income gap among academics and policy makers by exploring the root causes of gender inequality. In the following sections, we use a nationally representative data sample to test and analyze the above research framework and hypotheses.

\section{Data, variables, and methods}

\section{Data source}

The data used in this study were extracted from the 2013 Chinese General Social Survey (CGSS). ${ }^{6}$ The survey adopts a four-level stratified sampling scheme. A total of 480 villages or residential committees were selected from across the country. Twenty-five families were selected from each village or residential committee, and an adult over the age of 18 from each family was randomly chosen to complete an interview. The content of the survey, which is consistent with the goals of this study, covers social demographic attributes, labor incomes, and gender role attitudes.

Affected by the dualistic urban-rural employment system, labor force participation and income determination mechanisms of agricultural labor are significantly different from those of urban non-agricultural labor. Therefore, those currently working in agriculture and those not working and without non-agricultural work experience are excluded to improve the comparability of research objects. To be consistent with existing research, our sample is limited to the working population of 18-60 years of age, thus excluding those studying at school, unable to work, or retired. ${ }^{7}$

\footnotetext{
${ }^{6}$ The data used in this paper are from the Chinese General Social Survey (CGSS) project managed by the National Survey Research Center at Renmin University of China. Their provision of these data is appreciated. ${ }^{7}$ In China, the legal retirement age is different between men and women. For men, it is 60 . The age of retirement for women is 55 years of age. That for women at the county level, deputy county (section) level or equivalent section level, and deputy section level and for female professional technicians with senior professional posts is 60 years of age. We use the age of 60 for our data screening, but we also remove retired samples to control for the influence of retirement policies while keeping qualified samples.
} 


\section{Variables}

The annual income that individuals earn in the labor market is selected as our dependent variable and is a continuous variable measured by specific figures provided under "annual occupation/labor income" by the respondents. The variable is calculated as a logarithm so that it is close to a normal distribution. The questionnaire surveys the average number of hours worked per week, allowing individual hourly wages to be estimated. However, as the number of working hours is affected by gender role attitudes, we use annual income as our dependent variable to provide a comprehensive account of the influence of working hours on the gender income gap.

Gender role attitudes are used as our main explanatory variable. In the CGSS questionnaire, measurement items on gender role attitudes include "men are career oriented while women are family oriented," "men are born more capable than women," "having a good marriage is more important than having a good job," and "in an economic downturn, female employees should be fired first." These items reflect traditional views of gender roles, of women being inferior to men, of women's reliance on men, and of prioritizing men's access to employment opportunities, respectively. ${ }^{8}$ Respondents evaluate the above statements on a 5-point Likert scale with options "strongly disagree, " "disagree," "neutral," "agree," and "strongly agree."

To avoid collinearity among measurement indexes and ensure comparability between samples, the common average synthesis method is employed to obtain a comprehensive evaluation score for gender role attitudes. ${ }^{9}$ The higher the score, the more traditional gender role attitudes are. The reliability test shows that the alpha reliability of the evaluation score is 0.684 .

This paper controls for human capital and individual characteristic variables commonly used in income models. Human capital is measured by education level and health. According to classification options for education level included in the questionnaire, we operationalize education level as a set of dummy variables: junior high school and below (including no education), senior high school (including technical secondary school), junior college, and above. In the analysis, those with junior high school and below are used reference groups. Given the self-rated health conditions of the questionnaire, the variable of good health is constructed as a binary dummy variable whereby "very healthy," "relatively healthy," and "general" are assigned a value of 1 and "relatively unhealthy" and "very unhealthy" are assigned a value of 0 . Individual and family characteristics include age (continuous variable) and its square term ${ }^{10}$, marital status, and childbearing. Marital status is also measured as a group of dummy variables including unmarried, married, divorced, and widowed. Unmarried is used as the reference group in our analysis.

Occupational characteristics that affect income are controlled in the estimation and include the average number of hours worked per week and occupational status. Labor force participation and working hours are important influencing factors of income. We also recognize that gender role attitudes may affect individual income through working

\footnotetext{
${ }^{8}$ The correlation between these indicators is greater than 0.6. We did not use measurement items related to the division of housework, e.g., "a husband and wife should share housework equally" to evaluate gender role attitudes, as this variable is weakly correlated with the other indicators $(P=0.374)$.

${ }^{9}$ We used an exploratory factor analysis to test the robustness of our results and found that we could identify a common factor and obtain consistent regression analysis results.

${ }^{10}$ To improve the legibility of the regression coefficient, the square term of age is divided by 10.
} 
hours. We control for these factors. Some studies have noted the effect of occupational segregation on gender income stratification. As gender role attitudes affect both occupational choice and job promotion, the International Socio-Economic Index of Occupational Status (ISEI) is used as a continuous variable of 16 to 90 converted from respondents' occupation codes based on the International Standard Classification of Occupations (ISCO 88).

Other control variables used include institutional and structural factors. In Chinese society, the household registration system and party membership also affect social status and income. We thus control for demographic variables such as party membership and household registration in the income model. Party membership is also encoded as a binary variable with a value of 1 assigned for members of the Communist Party of China (CPC) and a value of 0 assigned for others. To identify differences between urban and rural household registration, we divide household registration statuses into urban and rural with rural residences assigned a value of 1 and nonrural residences assigned a value of 0 . Given that regional differences in levels of economic and social development and income also affect individual labor force participation and income, regional dummy variables for provinces are added to the model to control for the impacts of structural factors.

\section{Analytical strategy}

The data analysis presented this paper includes two parts. The first part involves exploring the impact of gender role attitudes on income and the gender income gap; the second part illustrates mechanisms of the influence of gender role attitudes on income.

It is worth noting that the model and regression methods are employed to explore the causal relationships between variables. It is usually necessary to consider or correct endogeneity caused by potential reverse causality and omitted variables. However, this necessity is not the case for the present study. On one hand, individual gender role attitudes are shaped in childhood or adolescence and are influenced by one's family of origin (Platt and Polavieja 2016), which occurs prior to acquiring labor market experience; on the other hand, gender role attitudes formed during childhood socialization form relatively stable value orientations. While not unchangeable, most people's gender attitudes remain stable even after experiencing life course events such as childbirth and postpartum employment (Schober and Scott 2012). Given these factors, it is reasonable to assume that gender role attitudes affect labor behavior and labor income rather than the reverse. ${ }^{11}$ It is thus appropriate to use a multiple linear regression analysis after controlling for most confounding variables.

To address potential third-party confounding factors such as omitted variables, we use an instrumental variable (IV). When data are available, the existing literature generally uses aggregate data of the community or regional level as an instrumental variable of individual-level indicators. We measure the average score for community-level gender role attitudes based on the initial CGSS sample (including the retired elderly) as an instrumental variable of individual gender role attitudes of the labor sample. According to socialization and formation mechanisms of gender role attitudes, norms

${ }^{11}$ The endogeneity test results also provide some evidence for the causal direction assumption. 
at the community level play a significant role in the formation of individual gender role attitudes, but they do not directly affect individual behaviors or labor incomes; in other words, they only affect individual labor behavior and labor income through individual gender role attitudes. In addition, the gender role attitudes of older generations at the community level affect those of younger generations, but the behaviors of younger generations, i.e., the working age population, will not in turn affect the gender role attitudes of the elderly. Therefore, there is a correlation between the instrumental variable and endogenous explanatory variables without reverse causality. The instrumental variable selected is thus reasonable to some extent.

Furthermore, since some respondents are not involved in labor employment and their labor income cannot be measured, values missing from the sample are thus not entirely random. Traditional gender role attitudes emphasizing "breadwinning men and homemaking women" may affect the labor force participation behaviors of the respondents and especially those of female respondents. Removing those have left the labor market and without occupation or wage information may lead to sample selection bias and underestimate the impact of gender role attitudes on labor income and the gender income gap. Therefore, Heckman's two-step method is adopted to correct for potential sample selection bias in the income model. In addition, in all regression analyses, aggregation effects of the samples at the township (subdistrict) level are adjusted to obtain the robust standard error.

\section{Results and discussion}

\section{Descriptive analysis}

Table 1 presents the means and distributions of independent and dependent variables by gender. The data show that gender role attitudes held by men and women in China reflect both traditional and modern views with an average score of 2.806 close to the average value of the scale. This outcome implies that traditional gender role attitudes in Chinese society and especially those of men have not changed substantially with modernization. Compared to women's, men's gender role attitudes tend to be more

Table 1 Summary statistics for variables

\begin{tabular}{lllll}
\hline Variables & Full sample & Male & Female & $\begin{array}{c}\text { Male-female } \\
\text { difference }\end{array}$ \\
\hline Logarithm of income & 10.204 & 10.332 & 10.007 & $.324^{* * *}$ \\
Gender role attitudes & 2.806 & 2.891 & 2.706 & $.185^{* * *}$ \\
Labor participation & .861 & .949 & .757 & $.192^{* * *}$ \\
Working hours per week & 40.371 & 45.986 & 33.755 & $12.230^{* * *}$ \\
ISEl & 41.258 & 40.553 & 42.088 & $-1.534^{* * *}$ \\
Education level & 1.818 & 1.848 & 1.782 & $.066^{* * *}$ \\
Good health & .943 & .940 & .947 & -.007 \\
Age & 38.816 & 39.996 & 37.425 & $2.570^{* * *}$ \\
Married & .819 & 0.800 & .842 & $-.041^{* * *}$ \\
Divorced/widowed & .040 & .037 & .044 & -.007 \\
CPC member & .114 & .147 & .075 & $.073^{* * *}$ \\
Rural household registration & .497 & .482 & .515 & $-.033^{* *}$ \\
\hline ***p<0.01, **p<0.05, and ${ }^{*} p<0.1$ & & & &
\end{tabular}


traditional (their indicator value is higher). This result is consistent with other research findings obtained from samples of different sizes in China. That is, women's gender role attitudes are more inclined to be progressive and focused on equality than men's (Liu and Tong 2014).

Women's disadvantages in terms of income levels are still obvious. Men's average incomes are roughly $32.4 \%$ higher than women's. At the same time, the female labor force participation rate is only $75.7 \%$ and significantly lower than the male rate (94.9\%). Correspondingly, women work fewer hours in the labor market, working an average of $12 \mathrm{~h}$ less per week than men. However, the occupational status (ISEI) of employed women seems to be slightly higher than that of men. ${ }^{12}$ The results shown in Table 1 also demonstrate that while women's education levels are constantly improving, average education levels among men are still higher than those among women. Moreover, the average age and proportion of CPC party members for the male sample are relatively high, and proportions of married individuals and levels of rural household registration are relatively low.

The results of our descriptive analysis reveal gendered differences in human capital and demographic characteristics such as education levels, which may have explanatory power in relation to the gender income gap. More importantly, we further explore gender role attitudes and their distinct impacts on men's and women's incomes. In the following section, while controlling for other influencing income factors, we examine and analyze whether and how gender role attitudes affect income.

\section{Regression results}

We first apply the ordinary least square method (OLS) and then address problems of sample selection bias and endogeneity to obtain robust causal relationships among variables. The regression results are reported in Table 2.

Empirical studies have consistently shown that the gender income gap constitutes a fundamental structure of inequality in China's labor market, which is further confirmed in this paper. Model 1 in Table 2 demonstrates that the incomes of female workers are still significantly lower than those of male workers under the same conditions even though differences in human capital and individual characteristics have been controlled. The gender gap is measured at roughly $39.4 \%$.

We adopt a new research design to incorporate gender role attitudes and their interaction item with gender to obtain complementary findings. The results of models 2 and 3 indicate that gender role attitudes have different impacts on the incomes of men and women. Traditional gender role attitudes are detrimental to women's earnings but do not affect men's average incomes. This result and its theoretical implications are consistent with trends found in the USA and other countries (Corrigall and Konrad 2007; Stickney and Konrad 2007).

As noted above, it is necessary to test for potential sample selection bias and endogeneity bias in a causal analysis to obtain robust and consistent estimation results. For the female sample, we adopt Heckman's two-step method to correct for sample selection bias. In the first stage model, the indicator variables are commonly used

\footnotetext{
${ }^{12}$ Since the sample leaving the labor force or unemployed does not have an occupational status index, to make up for lost information, we employed multiple imputation.
} 
Table 2 Impact of gender role attitudes on labor income

\begin{tabular}{|c|c|c|c|c|c|c|}
\hline \multirow[t]{2}{*}{ Variables } & \multicolumn{3}{|c|}{ Full sample } & \multicolumn{3}{|c|}{ Female subsample } \\
\hline & $\overline{\mathrm{M} 1}$ & $\mathrm{M} 2$ & M3 & M4-OLS & M5-Heckman & M6-IV \\
\hline Female & $-.394^{* * *}$ & $-.399^{* * *}$ & $-.170^{* *}$ & & & \\
\hline Gender role attitudes & & $-.028^{*}$ & .007 & $-.075^{* * *}$ & $-.076^{* * *}$ & $-.162^{* *}$ \\
\hline Female $\times$ gender role attitudes & & & $-.083^{* * *}$ & & & \\
\hline Senior high school & $.174^{* * *}$ & $.170^{* * *}$ & $.168^{* * *}$ & $.212^{* * *}$ & $.217^{* * *}$ & $.192^{* * *}$ \\
\hline College or higher & $.392^{* * *}$ & $.389^{* * *}$ & $.385^{* * *}$ & $.437^{* * *}$ & $.451^{* * *}$ & $.418^{* * *}$ \\
\hline Good health & $.307^{* * *}$ & $.309^{* * *}$ & $.304^{* * *}$ & $.367^{* * *}$ & $.375^{* * *}$ & $.363^{* * *}$ \\
\hline Age & $.075^{* * *}$ & $.076^{* * *}$ & $.077^{* * *}$ & $.073^{* * *}$ & $.078^{* * *}$ & $.075^{* * *}$ \\
\hline Age square/10 & $-.010^{* * *}$ & $-.010^{* * *}$ & $-.011^{* * *}$ & $-.010^{* * *}$ & $-.010^{* * *}$ & $-.010^{* * *}$ \\
\hline Working hours per week & $.003^{* * *}$ & $.003^{* * *}$ & $.003^{* * *}$ & $.004^{* * *}$ & $.004^{* * *}$ & $.004^{* * *}$ \\
\hline ISEl & $.009^{* * *}$ & $.009^{* * *}$ & $.009^{* * *}$ & $.010^{* * *}$ & $.010^{* * *}$ & $.009^{* * *}$ \\
\hline Married & $.175^{* * *}$ & $.177^{* * *}$ & $.175^{* * *}$ & .028 & .017 & .032 \\
\hline Divorced/widowed & $.145^{* *}$ & $.142^{* *}$ & $.141^{* *}$ & .100 & .098 & .093 \\
\hline CPC member & $.062^{*}$ & $.060^{*}$ & $.061^{*}$ & .027 & .031 & .018 \\
\hline Rural household registration & -.004 & -.001 & .001 & -.027 & -.027 & -.011 \\
\hline Heckman $\lambda$ & & & & & .056 & \\
\hline Hausman test & & & & & & 1.88 \\
\hline N & 4218 & 4218 & 4218 & 1666 & 2464 & 1666 \\
\hline
\end{tabular}

The omitted reference category for education level is junior high school or lower and that for marital status is unmarried. Dummy variables of the provincial level are controlled in all models. Standard errors are clustered by township (subdistrict)

${ }^{* * *} p<0.01,{ }^{* *} p<0.05$, and ${ }^{*} p<0.1$ (two-tailed test)

variables that measure factors that affect labor force participation, including gender role attitudes, education level, marital status, and whether an individual has children aged 6 and below. ${ }^{13}$ The regression results show that (model 5) the detrimental influence of traditional gender role attitudes on women's incomes remains robust with an additional 798 censored samples. Moreover, the absolute value of the regression coefficient is only slightly higher than the estimated coefficient obtained by the ordinary least square method (model 4). This result validates the inference that if those not participating in work and for whom income information cannot be obtained were removed, the effect of gender role attitudes may be underestimated due to sample selection, as gender role attitudes affect labor force participation. However, a Wald statistical test shows that the difference in the regression coefficient is not significant $\left(\chi^{2}=1.46, p=0.227\right)$, indicating that the negative effect of gender role attitudes on women's labor incomes is not caused by sample selection.

Furthermore, average scores for gender role attitudes at the community level are used as an instrumental variable for individual gender role attitudes (IV). We find that the instrumental variable passes the weak instrumental variable test $(F=179.968, p=0.000)$. The regression results of the first stage demonstrate that the instrumental variable is highly correlated with individual gender role attitudes, for which the regression coefficient is $0.817(p=0.000)$. The results of the instrumental variable regression model (model 6 in Table 2) shown in Table 3 demonstrate that traditional gender role attitudes still have a

\footnotetext{
${ }^{13}$ The regression results of the first selection equation are discussed in the mechanism analysis below. See the second column of Table 4.
} 
Table 3 First stage results for instrumental variable regression

\begin{tabular}{llll}
\hline Variables & Beta & Robust standard errors & Sig. \\
\hline Average gender role attitudes & 0.817 & 0.061 & 0.000 \\
Senior high school & -0.209 & 0.048 & 0.000 \\
College or higher & -0.169 & 0.060 & 0.005 \\
Good health & -0.054 & 0.101 & 0.598 \\
Age & 0.024 & 0.015 & 0.101 \\
Age square/10 & -0.002 & 0.002 & 0.390 \\
Working hours per week & -0.001 & 0.001 & 0.517 \\
ISEl & -0.002 & 0.001 & 0.106 \\
Married & 0.003 & 0.070 & 0.969 \\
Divorced or widowed & -0.075 & 0.103 & 0.464 \\
CPC member & -0.127 & 0.060 & 0.036 \\
Rural household registration & 0.111 & 0.046 & 0.016 \\
Adjusted $R$-squared & 0.222 & & \\
$N$ & 1666 & & \\
\hline Dummy variables of the provincial level are controlled. Standard errors are clustered by township (subdistrict) &
\end{tabular}

significant negative effect on women's incomes and that the absolute value of the estimation coefficient of the instrumental variable is larger than that of the OLS estimation. This outcome is the case because the instrumental variable regression reflects the local average treatment effect, i.e., the effect of the part greatly affected by community social norms. These people may be inclined to conform to cultural norms and social expectations, causing gender role attitudes to have a stronger impact on them. This phenomenon helps us understand why the regression coefficient of the instrumental variable is larger than that of the OLS model. However, the results of Hausman's endogeneity test indicate that the null hypothesis cannot be rejected, i.e., there is no systematic discrepancy between the OLS model and the instrumental variable model $\left(\chi^{2}=1.88, p=1.000\right)$. This lack of discrepancy confirms that the regression model does not show serious signs of endogeneity such as reverse causality and omitted variable bias, and the OLS regression results are reliable. Gender role attitudes form in early stages of the life course before entering the labor market, forming a set of relatively stable views and values. Moreover, our research objects are adults whose ideas and opinions on gender have long been "internalized in the heart and externalized in the line" and are thus difficult to change. Therefore, our theoretical and statistical results support the conclusion that individual gender role attitudes are exogenous.

Overall, the results of the ordinary least square regression and instrumental variable regression (see Table 2) show that traditional gender role attitudes have a significantly negative impact on women's incomes but have no significant effects on men's incomes. ${ }^{14}$ Therefore, hypothesis 1 is supported by statistical and theoretical evidence.

Moreover, consistent with existing research, the regression results given in Table 2 demonstrate that human capital indexes, e.g., education level and health, have a positive effect on income. Income and age also exhibit an inverted U-shaped relation. Average

\footnotetext{
${ }^{14} \mathrm{We}$ also use an instrumental variable regression to test the male sample. The results are consistent with the OLS results in that gender role attitudes have no significant impacts on men's wages.
} 
weekly working hours and occupational status have positive effects on the incomes of men and women. However, party membership seems to only increase the incomes of men and not those of women. Similarly, male workers obtain a positive wage premium through marriage while women's incomes are not significantly related to marital status.

The above results provide new empirical evidence of the cause of the gender income gap from the perspective of deep-seated cultural norms. Since the regression model shown in Table 2 controls individual human capital and other variables that affect labor income, the gender income gap reflected by the regression coefficient of the female dummy variable constitutes the part of influence of individual characteristics that cannot be observed. The change in the regression coefficient indicates that the absolute value of the regression coefficient of the female sample of model 3 is much lower than that of model 1, which implies that the unexplained part of the gender income gap is greatly reduced when interaction effects of gender role attitudes and the female dummy variable are included. In this sense, gender role attitudes have remarkable power in explaining the gender income gap.

Figure 2 offers a straightforward illustration of the impact of gender role attitudes on the gender income gap. Based on the regression equation of model 3, after controlling for other influential factors, gender role attitudes have a significantly different impact on the incomes of men and women. Figure 2 shows that when gender role attitudes shift from modern to traditional (from left to right), there is no significant change in the incomes of men, but women's incomes decrease, creating an expanding income gap between men and women. Taking extreme values at both ends as examples, the incomes of women with extremely traditional attitudes are 58.4\% lower than those of their male counterparts while those of women with progressive gender role attitudes are only $25.3 \%$ lower than those of their male counterparts. It can thus be inferred that the construction of egalitarian attitudes is greatly conducive to narrowing the income gap between men and women and to promoting women's development and gender equality.

To verify the impact of gender role attitudes on income stratification and to quantify its explanatory power and mechanisms, we adopt full-sample Oaxaca-Blinder

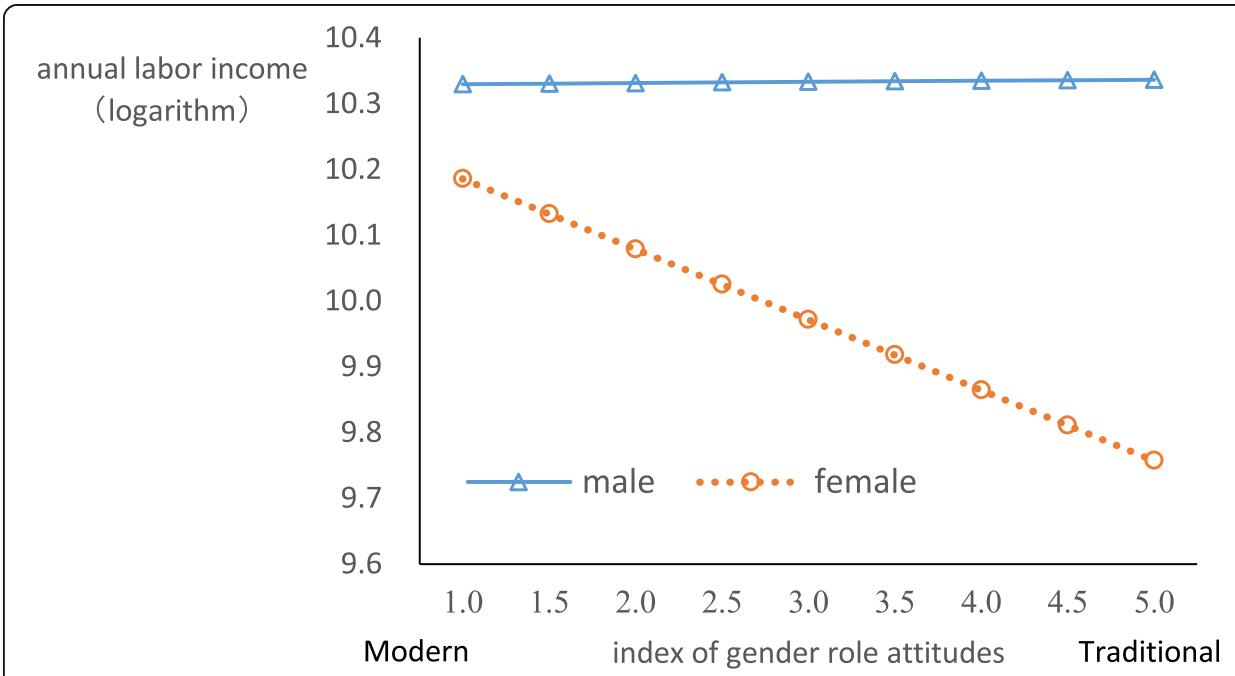

Fig. 2 The interaction effect of gender and gender role attitudes 
decomposition, the latest method developed to solve problems of wage standard selection and to incorporate gender dummy variables (see Fortin et al. 2011), to reveal the root cause of the gender income gap. As expected, the decomposition results demonstrate that gender role attitudes and especially their different effects on gendered incomes are central to the formation of the gender income gap. In other words, the impact of gender role attitudes on the gender income gap mainly derives from the regression coefficient differences. The influence of gender role attitudes accounts for $60.8 \%$ of the unexplainable part of the gender income gap (from regression coefficient differences). If the impact of gender role attitudes on the incomes of men and women tends to be the same, i.e., the negative effect of traditional gender role attitudes on female incomes decreases, and the income gap between men and women greatly reduced. Therefore, to eliminate the negative influence of traditional gender role attitudes and narrow the income gap between men and women, it is necessary to not only construct egalitarian attitudes but also apply effective interventions to reduce constraints and restrictions on women brought about by traditional gender role attitudes.

Before exploring how gender role attitudes affect incomes, we also examine which dimensions of gender role attitudes affect incomes. To avoid multicollinearity, we individually incorporate four different dimensions of gender role attitudes and their interaction items with the female variable into the regression model. Each dimension is coded as a dummy variable where "agree" and "strongly agree" responses are coded as 1 (traditional tendency), and other responses are coded as 0 . The regression results of models 7 to 10 in Table 4 indicate that when all other variables are controlled, only the coefficient of the first interaction item is statistically significant; that is, traditional views of "breadwinning men and homemaking women" have a significantly negative effect on women's incomes. For other dimensions of gender role attitudes, e.g., "men are born stronger than women," "a good marriage is more important than a good job," and "in an economic downturn, female employees should be fired first," regression coefficients of corresponding dummy variables and their interaction terms are not significant.

Therefore, compared to other dimensions of gender role attitudes, the traditional view of family breadwinners and caregivers forms the basis of gender role attitudes, reflecting the effect of social and cultural norms on the gender income gap. Meanwhile,

Table 4 Impact of specific gender role attitudes on labor income

\begin{tabular}{|c|c|c|c|c|}
\hline Variables & M7 & M8 & M9 & M10 \\
\hline Female & $-.324^{* * *}$ & $-.373^{* * *}$ & $-.374^{* * *}$ & $-.389 * * *$ \\
\hline Breadwinning men and homemaking women & .036 & & & \\
\hline Female $\times$ breadwinning men and homemaking women & $-.137^{* * *}$ & & & \\
\hline Men are more capable than women & & -.001 & & \\
\hline Female $\times$ men are more capable than women & & -.063 & & \\
\hline A good marriage is more important than a good job & & & .024 & \\
\hline $\begin{array}{l}\text { Female } \times \text { a good marriage is more important than a } \\
\text { good job }\end{array}$ & & & -.050 & \\
\hline Women should be fired first & & & & -.009 \\
\hline Female $\times$ women should be fired first & & & & -.067 \\
\hline N & 4218 & 4212 & 4215 & 4204 \\
\hline
\end{tabular}

Other control variables are the same as those used in Table 2. Standard errors are clustered by township (subdistrict) ${ }^{* * *} p<0.01,{ }^{* *} p<0.05$, and ${ }^{*} p<0.1$ (two-tailed test) 
hypothesis 1 is supported again in that traditional gender role attitudes mainly affect women's social behaviors but have no significant impact on men.

\section{Mechanism and mediating effect analysis}

The results discussed in above sections show that traditional gender role attitudes have a directly negative effect on women's incomes only when productivity characteristic variables are controlled. Meanwhile, consistent with existing studies, education level, labor force participation, working hours, and occupational status affect the gender income gap. In existing research, the part that can be explained by these factors is usually considered "reasonable." However, it is uncertain whether these factors are shaped by unreasonable social structures and cultural norms. Therefore, with the above analysis framework, we explore how and through which channel gender role attitudes affect women's incomes to test hypothesis 2 .

Since the mediating variables mentioned above are different types of dependent variables, we adopt the corresponding model and make relevant adjustments to the control variables. Like the continuous variable of occupational status (ISEI), education level is estimated by multiple linear regression. Since labor force participation is a dummy variable ${ }^{15}$, the Probit model and corresponding IV-Probit model are adopted. In addition, Tobit and IV-Tobit models are employed to analyze working hours.

However, consistent with the above findings, the average score of community gender role attitudes, which is an instrumental variable, passes the weak instrumental variable test. From our test of endogeneity, however, we find that we cannot reject the null hypothesis that individual gender role attitudes are exogenous. Therefore, the results of the two methods are consistent, and more effective multiple regression analysis results are preferred and reported (see Table 5).

For the model for education attainment, we follow practices described in $\mathrm{Wu}$ (2012) and other similar studies and control family of origin background variables, including the father's occupational status, whether the mother has received higher education or not, and dummy variables for household registration and ethnicity, to investigate differences in educational attainment between urban and rural areas and among different ethnicities. The regression results for education level given in the first column of Table 5 demonstrate that traditional gender role attitudes are negatively correlated with women's education levels. This finding is consistent with those of similar studies conducted on other countries (see Kosteas 2013). In addition, fathers' occupational statuses and mothers' education levels have significantly positive effects in promoting women's education attainment while women in urban areas complete more education that those in rural areas. These results show that while in today's market-oriented society women have achieved great progress in labor force participation and occupational status, traditional gender role attitudes continue to have a negative influence on women's expectations and investments in education. Given the analytical results above (Table 2) and as education level is a vital factor in determining individual income levels, it can be inferred that traditional gender role attitudes can reduce women's incomes and expand the income gap between men and women by inhibiting women's education levels. The

\footnotetext{
${ }^{15}$ Exiting the labor market is defined as being unemployed and "not looking for a job" over the past 3 months, i.e., no labor participation behavior (coded as 0). Those employed and unemployed but seeking work are defined as participants in the labor market (coded as 1).
} 
Table 5 Mechanisms of gender role attitude effects on women's income

\begin{tabular}{|c|c|c|c|c|}
\hline \multirow[t]{2}{*}{ Variables } & Education attainment & Labor force participation & Working hours & ISEI \\
\hline & OLS & Probit & Tobit & OLS \\
\hline Gender role attitudes & $-.133^{* * *}$ & $-.171^{* * *}$ & $-3.666^{* * *}$ & $-.835^{* * *}$ \\
\hline Senior high school & & .074 & -.726 & $6.213^{* * *}$ \\
\hline College or higher & & $.813^{* * *}$ & .878 & $15.040^{* * *}$ \\
\hline Good health & & $.418^{* * *}$ & $13.390^{* * *}$ & $2.411^{* * *}$ \\
\hline Age & $-.030^{* * *}$ & $.154^{* * *}$ & $4.844^{* * *}$ & $.493^{* * *}$ \\
\hline Age square/10 & .001 & $-.019^{* * *}$ & $-.585^{* * *}$ & $-.073^{* * *}$ \\
\hline Married & & $-.607^{* * *}$ & $-12.614^{* * *}$ & -.247 \\
\hline Divorced or widowed & & -.219 & -5.046 & .374 \\
\hline Children under 6 years of age & & $-.564^{* * *}$ & $-12.051^{* * *}$ & \\
\hline CPC member & & $.323^{* *}$ & 1.795 & $5.444^{* * *}$ \\
\hline Rural household registration & $-.620^{* * *}$ & .009 & $3.391^{*}$ & $-2.499^{* * *}$ \\
\hline Han & .043 & & & \\
\hline ISEl of father & $.009^{* * *}$ & & & \\
\hline Mother with higher education & $.290^{* * *}$ & & & \\
\hline$N$ & 2189 & 2465 & 2465 & 2465 \\
\hline
\end{tabular}

Dummy variables of the provincial level are controlled. Standard errors are clustered by township (subdistrict) ${ }^{* * *} p<0.01,{ }^{* *} p<0.05$, and ${ }^{*} p<0.1$ (two-tailed test)

mediating mechanism of hypothesis $2 \mathrm{a}$ is thus supported. Conversely, this outcome implies that the development of norms focused on gender equality could elevate women's educational attainment and increase their income levels.

Similarly, after controlling for education level and individual and family structure variables, traditional gender role attitudes are found to not only hinder women's labor force participation but also reduce their average weekly working hours (see regression results for labor force participation and working hours in Table 5). It may be that women with traditional gender role views are burdened with more family responsibilities and spend less time and energy in the labor market when work-family role conflicts exist. In this sense, we prove that traditional gender role attitudes still have strong effects. Gender norms not only refer to individuals' views and values but are also embodied in the regulation and guiding of specific behaviors in individual daily life. In other words, the influence of traditional gender norms on gender structures is not only realized through the cultivation of ideas but also reflected through behaviors by means of attitudes, ultimately leading to gender inequality. Given the positive impacts of labor force participation and working hours on income levels (see Table 2), these results demonstrate that progressive gender role attitudes can narrow the gender income gap by promoting women's labor force participation. Otherwise, the income gap between men and women should continue to expand. The mediating mechanism of hypothesis $2 \mathrm{~b}$ is thus verified.

Meanwhile, the regression results for occupational status given in Table 5 indicate that gender role attitudes are negatively correlated with occupational status. The occupational statuses of women with traditional gender role attitudes are lower than those of women with progressive gender attitudes. In other words, progressive gender norms are conducive to women's career development while traditional cultural norms are not conducive to the promotion of women's occupational status. The mediating mechanism 
of hypothesis $2 \mathrm{c}$ is thus verified and supported. This finding is consistent with existing empirical studies showing that women are concentrated in low-level "feminized" positions and that discrimination that women encounter in terms of job promotion widens the average income gap between men and women (Qing and Zheng 2013). However, relative to the theory of gender discrimination in the labor market, traditional gender role attitudes have greater explanatory power in revealing the source of gender inequality. As gender role attitudes have significant effects on so-called achieved factors such as education levels, a large proportion of the gender income gap explained by these variables may be a result of gender role attitudes. Therefore, values estimated under the control of human capital and individual characteristic variables are far lower than the actual impacts of gender role attitudes on the gender income gap. Rather, we cannot fully understand the root causes of gender inequality when we regard differences in human capital, e.g., education attainment, as an established fact and base causes of the gender income gap on this assumption rather than investigating why these factors are different.

In sum, the mediating mechanism of hypothesis 2 is supported by evidence from national representative samples. Our analytical results indicate that gender inequality is not merely the result of biological differences or a consequence of individual rational decision-making but a product of profound social and cultural processes. In fact, the influence of gender role attitudes on the income gap between men and women is the combined result of interactions of several factors. During socialization, every individual learns and conforms to the norms and expectations of gender roles; develops and forms preferences and values that affect individual behavior; and thus, shapes his or her own behavior through interactions with family members, peers, and the mass media. In traditional societies based on patriarchy and patrilocal residence, a woman is required to stay at home and take care of her husband and children without appearing in public, leaving no corresponding market return for education investment among women. Women who adhere to traditional gender role attitudes, e.g., "breadwinning men and homemaking women," not only exhibit less initiative to participate in the labor market but also limit their labor force participation rates in the midst of objective conditions of insufficient education. At the same time, to meet social norm and gender role expectations, women must bear more responsibility for housework and family caregiving, causing the roles of modern professional women to conflict with traditional roles of being good wife and mother. In interactions between individuals, families, and the market, women's working hours and energy spent in the labor market are limited, which is not conducive to women's occupational status and income growth.

\section{Concluding remarks}

In reference to institutional and cultural transformation, this paper provides a more complete account of whether and how gender role attitudes affect individual incomes and the gender income gap while providing a new perspective for further understanding causes of the gender income gap.

The results show that gender role attitudes have a salient impact on the income gap between men and women. Compared to those of women, the gender role attitudes of men tend to be more traditional. When other factors are controlled, traditional gender role attitudes have a significantly negative impact on women's earning and have no 
significant effect on men's average incomes. After combining gender role attitudes and their interaction item with the gender variable, the absolute value of the regression coefficient of the dummy variable for women drops significantly, indicating that traditional gender role attitudes play important roles in explaining the gender income gap. The decomposition results further demonstrate that gender role attitudes can explain $60 \%$ of the proportion that cannot be explained by differences in productivity characteristics.

In addition, traditional gender role attitudes have a significantly negative correlation with women's education levels, labor force participation rates, working hours, and occupational status. Traditional gender role attitudes reduce the incomes of women through these mediating factors and thus expand the gender income gap. This outcome implies that traditional gender role attitudes are deeply rooted in gender inequality in the realm of income distribution, which has a profound and invisible impact. It is so common for women's incomes to be lower than men's that we almost forget that this pattern is a reflection of gender discrimination caused by the values of men and women.

This paper expands the research paradigm of human capital and provides a new perspective deepening understanding of the source and persistence of the gender income gap. The existing research has tended to attribute the gender income gap to differences in human capital or gender segregation, although scholars also show that these conventional factors cannot explain the root causes shaping gender income gap. With economic and social development and changes in family structures, traditional norms have not declined or disappeared, and cultural norms and social practices focused on "breadwinning men and homemaking women" have not changed substantially according to our results. However, some studies show that patriarchal norms are declining while the educational status and decision-making power of women are rising. Moreover, gender role attitudes are often transferred from one generation to the next under the guidance of parents' words and deeds (Qing 2018). In other words, generational change may not be able to change traditional roles and gender structures. Therefore, if norms of gender equality do not emerge and play a substantial role, even if women's education levels catch up with or even exceed those of men, women's income disadvantages may remain.

Meanwhile, this paper provides empirical evidence for how gender role attitudes influence women's education attainment, labor force participation, occupational status, and working hours in the labor market, which is helpful in comprehending the farreaching effects of traditional cultural norms on individual decision-making and sources of gender inequality and in constructing follow-up research. However, due to the limitations of data and analysis methods, these impacts cannot be comprehensively evaluated. For example, society expects women to be gentle and conservative and men to be confident and decisive (Eagle 1987), leading to the formation of gendered differences in psychological characteristics, e.g., risk aversion and competition awareness through individual socialization, and ultimately inducing different market behaviors and income inequality. Further research must consider more mediating mechanisms in the analysis framework.

The present study also has instructive implications for the top-level design of China's gender equality policies. Traditional gender role attitudes have deep historical roots and a strong foundation, which are closely related to market transformation and 
implementation effects of gender equality policies. In this sense, the support of national systems and policies should run parallel with changes in culture. In recent years, the state has continuously consolidated the institutional construction of policies that promote gender equality and protect women and that emphasize equal rights for women under law and oppose all forms of gender discrimination. However, the promotion of women's development and gender equality has never been achieved through the use of slogans alone. Central to gender equality and an advanced gender culture is the effective implementation and operation of specific policies. Therefore, it is necessary to develop a further understanding of the socioeconomic and psychological basis on which traditional gender role attitudes have been built to take targeted measures to raise awareness of gender equality and to promote women's active participation in the construction and sharing of economic and social development.

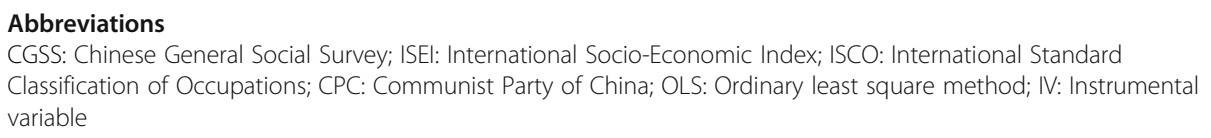

\section{References}

Akerlof, George A., and Rachel E. Kranton. 2000. Economics and Identity. The Quarterly Journal of Economics 115 (3): $715-753$. Alesina, Alberto, Giuliano Paola, and Nunn Nathan. 2013. On the Origins of Gender Roles: Women and the Plough. The Quarterly Journal of Economics 128 (2): 469-530.

Bertrand, Marianne. 2011. New Perspectives on Gender. In Ashenfelter and Card, ed. Handbook of Labor Economics, 4:15431590. North Holland: Elsevier.

Bertrand, Marianne, Kamenica Emir, and Jessica Pan. 2015. Gender Identity and Relative Income within Households. The Quarterly Journal of Economics 130 (2): 571-614.

Blau, Francine D., and Lawrence M. Kahn. 2017. The Gender Wage Gap: Extent, Trends, and Explanations. Journal of Economic Literature 55 (3): 789-865.

Bursztyn, Leonardo, Fujiwara Thomas, and Pallais Amanda. 2017. 'Acting Wife': Marriage Market Incentives and Labor Market Investments. American Economic Review 107 (11): 3288-3319.

Christie-Mizell, C. Andr. 2006. The Effects of Traditional Family and Gender Ideology on Earnings: Race and Gender Differences. Journal of Family \& Economic Issues 27 (1): 48-71.

Christie-Mizell, C. André, Jacqueline M. Keil, Kimura Aya, and Stacye A. Blount. 2007. Gender Ideology and Motherhood: The Consequences of Race on Earnings. Sex Roles 57 (9): 689-702.

Correll, Shelley. 2004. Constraints into Preferences: Gender, Status, and Emerging Career Aspirations. American Sociological Review 69 (1): 93-113.

Corrigall, Elizabeth, and Alison Konrad. 2007. Gender Role Attitudes and Careers: A Longitudinal Study. Sex Roles 56 (11-12): 847-855.

Davis, Shannon N., and Lisa D. Pearce. 2007. Adolescents' Work-Family Gender Ideologies and Educational Expectations. Sociological Perspectives 50 (2): 249-271.

Diekman, Amanda B., Elizabeth R. Brown, Amanda M. Johnston, and Emily K. Clark. 2010. Seeking Congruity Between Goals and Roles: A New Look at Why Women Opt Out of Science, Technology, Engineering, and Mathematics Careers. Psychological Science 21 (8): 1051-1057.

Eagly, Alice H. 1987. Sex Differences in Social Behavior: A Social-Role Interpretation. Hillsdale, NJ: Lawrence Erlbaum Associates. Eagly, Alice H., and Steven J. Karau. 2002. Role Congruity Theory of Prejudice Toward Female Leaders. Psychological Review 109 (3): 573-598. 
Eagly, Alice H., and Wendy Wood. 1999. The Origins of Sex Differences in Human Behavior: Evolved Dispositions Versus Social Roles. American Psychologist 54 (6): 408-423.

Firestone, Juanita M., Richard J. Harris, and Linda C. Lambert. 1999. Gender Role Ideology and the Gender Based Differences in Earnings. Journal of Family \& Economic Issues 20 (2): 191-215.

Fortin, Nicole M. 2015. Gender Role Attitudes and Women's Labor Market Participation: Opting-Out, AIDS, and the Persistent Appeal of Housewifery. Annals of Economics \& Statistics 117-118: 379-401.

Fortin, Nicole, Lemieux Thomas, and Firpo Sergio. 2011. Decomposition Methods in Economics. In Ashenfelter and Card, ed. Handbook of Labor Economics,1-102. North Holland: Elsevier.

Gutek, Barbara A., Searle Sabrina, and Klepa Lilian. 1991. Rational versus Gender Role Explanations for Work-Family Conflict. Journal of Applied Psychology 76 (4): 560-568.

Hansen, Casper Worm, Jensen Peter Sandholt, and Skovsgaard Christian Volmar. 2015. Modern Gender Roles and Agricultural History: The Neolithic Inheritance. Journal of Economic Growth 20 (4): 365-404.

Haveman, Heather A., and Lauren S. Beresford. 2012. If You're So Smart, Why Aren't You the Boss? Explaining the Persistent Vertical Gender Gap in Management. The ANNALS of the American Academy of Political and Social Science 639 (1): 114-130.

He, Guangye, and Xiaogang Wu. 2015. Marketization, Economic Development and Gender Earnings Inequality in Urban China. Sociological Studies 1: 140-165.

Jayachandran, Seema. 2015. The Roots of Gender Inequality in Developing Countries. Annual Review of Economics 7 (1): 63-88.

Jia, Yunzhu, and Dongling Ma. 2015. Changes in a Gender Perspective from Multifaceted Perspective: The Case with "Men Dominating the Outs While Women Dominating the Inside of Household". Collection of Women's Studies 3: 29-36.

Judge, Timothy, and Beth Livingston. 2008. Is the Gap More than Gender? A Longitudinal Analysis of Gender, Gender Role Orientation, and Earnings. Journal of Applied Psychology 93 (5): 994-1012.

Kosteas, Vasilios. 2013. Gender Role Attitudes, Labor Supply, and Human Capital Formation. Industrial Relations 52 (4): 915-940.

Li, Chunling, and Shi Li. 2008. Rising Gender Income Gap and Its Dynamics in China: Market competition or sex discrimination? Sociological Studies 2: 94-117.

Li, Shi, Song Jin, and Liu Xiaochuan. 2014. Evolution of the Gender Wage Gap among China's Urban Employees. Management World 3: 53-65.

Lips, Hilary. 2013. The Gender Pay Gap: Challenging the Rationalizations. Perceived Equity, Discrimination, and the Limits of Human Capital Models. Sex Roles 68 (3): 169-185.

Liu, Aiyu, and Xin Tong. 2014. The Present Situation of Gender Attitudes and the Factors Influencing Them: Based on the Third Survey of Women's Social Status in China. Social Sciences in China 2: 116-129.

Liu, Aiyu, Tong Xin, and Fu Wei. 2015. Household Division of Housework for Double-Income Family: Economic Dependence, Gender Ideologies, or Emotional Express? Chinese Journal of Sociology 2: 109-136.

Okamoto, Dina, and Paula England. 1999. Is There a Supply Side to Occupational Sex Segregation? Sociological Perspectives 42 (4): 557-582.

Platt, Lucinda, and Javier Polavieja. 2016. Saying and Doing Gender: Intergenerational Transmission of Attitudes towards the Sexual Division of Labour. European Sociological Review 32 (6): 820-834.

Qing, Shisong. 2017. Gender Role Attitudes, Family Responsibilities and Female-male Labor Force Participation Patterns. Journal of Social Sciences 11: 91-100.

Qing, Shisong. 2018. Intergenerational Transmission of Gender Role Attitudes in China. Chinese Journal of Population Science 6: 80-91.

Qing, Shisong, and Jiamei Zheng. 2013. Unequal Pay or Unequal Job: Effect of Hierarchical Segregation on Gender Earning Differentials. China Economic Quarterly 2: 735-756.

Schober, Pia, and Jacqueline Scott. 2012. Maternal Employment and Gender Role Attitudes: Dissonance Among British Men and Women in the Transition to Parenthood. Work, Employment \& Society 26 (3): 514-530.

Sinclair, Samantha, and Rickard Carlsson. 2013. What Will I Be When I Grow Up? The Impact of Gender Identity Threat on Adolescents' Occupational Preferences. Journal of Adolescence 36 (3): 465-474.

Song, Shaopeng. 2012. From Visible to Invisible: Housework in the Collective Period. Jiangsu Social Sciences 1: 116-125.

Stickney, Lisa T., and Alison Konrad. 2007. Gender-Role Attitudes and Earnings: A Multinational Study of Married Women and Men. Sex Roles 57 (11): 801-811.

Tong, Xin, and Aiyu Liu. 2015. A Model of Conjugal Cooperation in Housework for Urban Dual-career Couples - Based on the Third Survey of Women's Status in China (2010). Social Sciences in China 6: 96-111.

van der Vleuten, Maaike, Jaspers Eva, Maas Ineke, and Tanja van der Lippe. 2016. Boys' and Girls' Educational Choices in Secondary Education. The Role of Gender Ideology. Educational Studies 42 (2): 181-200.

Wang, Tianfu, Lai Yangen, and Li Bobai. 2008. Gender Income Gap under Redistribution and Its Evolution: 1995-2003. Sociological Studies 2: 23-53.

Wu, Xiaoying. 2009. The Transformation of Gender Discourse in the Context of Marketization. Social Sciences in China 2: 163-176.

Wu, Yuxiao. 2012. Gender Gap in Educational Attainment in Urban and Rural China. Chinese Journal of Sociology 4: 112-137.

Wu, Yuxiao, ans Wu, and Xiaogang. 2009. Occupational Gender Segregation and Gender Wage Gap in Urban China. Sociological Studies 4: 88-111.

Yang, Juhua. 2014. Continuity and Strategy: A Gender Pattern of Household Work Division in China between 1990-2010. Academic Research 2: 31-41.

Yang, Juhua. 2017. The Continuity and Change of Chinese Gender Norms in the Past 20 Years. Shandong Social Sciences 11: 60-71.

Zheng, Jiamei, and Shisong Qing. 2016. Non-Cognitive Skills, Psychological Characteristics and Gender Wage Gap. Economic Perspectives 7: 135-145.

Zuo, Jiping. 2005. Women's Liberation and Gender Equality in the 1950s: Experience and Interpretation of Urban Married Couples in China. Society 1: 182-209.

\section{Publisher's Note}

Springer Nature remains neutral with regard to jurisdictional claims in published maps and institutional affiliations. 